\title{
Charles TAYLOR
}

Philosophe québécois, professeur émérite de science politique et de philosophie à l’Université McGill, Montréal.

(1994)

\section{"Le pluralisme et le dualisme.”}

Un document produit en version numérique par Pierre Patenaude, bénévole, Professeur de français à la retraite et écrivain

Chambord, Lac-St-Jean.

Courriel: pierre.patenaude@gmail.com

Page web dans Les Classiques des sciences sociales.

Dans le cadre de la bibliothèque numérique: "Les classiques des sciences sociales" Site web: http://www.uqac.ca/Classiques_des_sciences_sociales/

Une bibliothèque développée en collaboration avec la Bibliothèque

Paul-Émile-Boulet de l'Université du Québec à Chicoutimi

Site web: http://bibliotheque.uqac.uquebec.ca/index.htm 


\section{Politique d'utilisation de la bibliothèque des Classiques}

Toute reproduction et rediffusion de nos fichiers est interdite, même avec la mention de leur provenance, sans l'autorisation formelle, écrite, du fondateur des Classiques des sciences sociales, Jean-Marie Tremblay, sociologue.

Les fichiers des Classiques des sciences sociales ne peuvent sans autorisation formelle:

- être hébergés (en fichier ou page web, en totalité ou en partie) sur un serveur autre que celui des Classiques.

- servir de base de travail à un autre fichier modifié ensuite par tout autre moyen (couleur, police, mise en page, extraits, support, etc...),

Les fichiers (.html, .doc, .pdf, .rtf, .jpg, .gif) disponibles sur le site Les Classiques des sciences sociales sont la propriété des Classiques des sciences sociales, un organisme à but non lucratif composé exclusivement de bénévoles.

Ils sont disponibles pour une utilisation intellectuelle et personnelle et, en aucun cas, commerciale. Toute utilisation à des fins commerciales des fichiers sur ce site est strictement interdite et toute rediffusion est également strictement interdite.

L'accès à notre travail est libre et gratuit à tous les utilisateurs. C'est notre mission.

Jean-Marie Tremblay, sociologue

Fondateur et Président-directeur général, LES CLASSIQUES DES SCIENCES SOCIALES. 
Cette édition électronique a été réalisée par Pierre Patenaude, bénévole, professeur de français à la retraite et écrivain,

Courriel : pierre.patenaude@gmail.com

à partir du livre, sous la direction de :

\section{Charles TAYLOR}

\section{"Le pluralisme et le dualisme.”}

Un texte publié dans l'ouvrage sous la direction d'Alain-G. Gagnon, Québec : État et société. Tome I., chapitre 3, pp. 61-84. Montréal : Les Éditions Québec/Amérique, 1994, 509 pp. Collection : Société : dossiers documents.

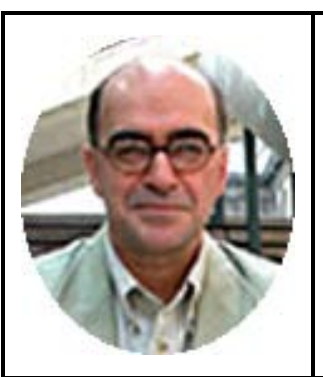

M Alain-G. Gagnon, politologue, professeur au département de sciences politique, UQÀM, nous a accordé le 17 mars 2006 son autorisation de diffuser électroniquement toutes ses publications dans Les Classiques des sciences sociales.

Courriel : gagnon.alain@uqam.ca

Polices de caractères utilisée :

Pour le texte: Times New Roman, 12 points.

Pour les notes de bas de page : Times New Roman, 10 points.

Édition électronique réalisée avec le traitement de textes Microsoft Word 2008 pour Macintosh.

Mise en page sur papier format : LETTRE US, 8.5' x 11'”.

Édition numérique réalisée le 21 août 2012 à Chicoutimi, Ville de Saguenay, Québec.

\section{f. Fait avec}




\section{Charles TAYLOR}

Philosophe québécois, professeur émérite de science politique et de philosophie à l’Université McGill, Montréal.

\section{"Le pluralisme et le dualisme.”}

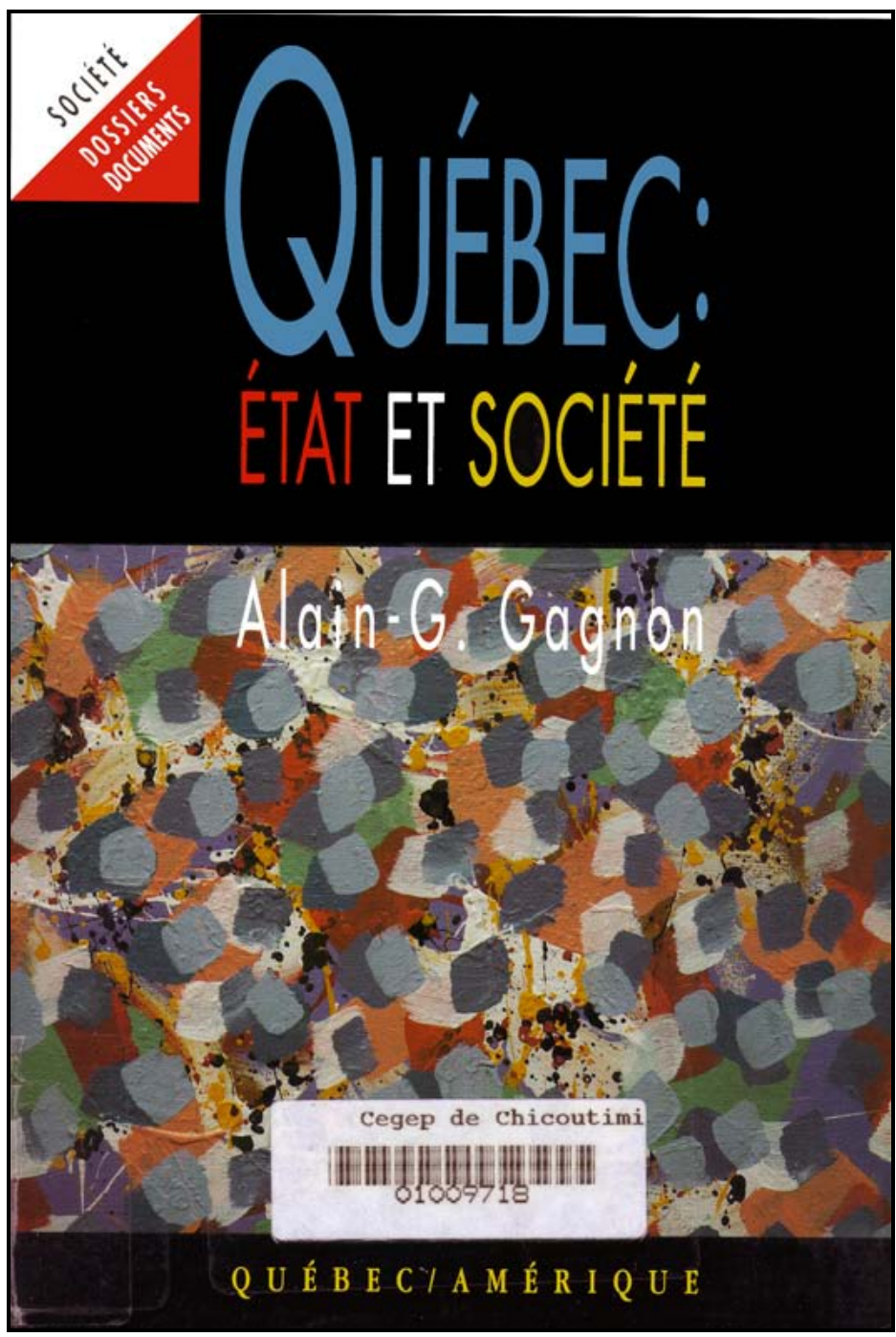

Un texte publié dans l'ouvrage sous la direction d'Alain-G. Gagnon, Québec : État et société. Tome I., chapitre 3, pp. 61-84. Montréal : Les Éditions Québec/Amérique, 1994, 509 pp. Collection : Société : dossiers documents. 
[17]

\author{
Québec : État et société. Tome I. \\ NOTES SUR \\ LES COLLABORATEURS
}

\title{
Charles Taylor
}

Charles Taylor est professeur titulaire de philosophie à l'Université McGill. Ses recherches portent sur la pensée allemande, l'épistémologie et l'interprétation de l'identité moderne. Il est l'auteur de Grandeur et misère de la modernité (1992) Rapprocher les solitudes (1992), et Multiculturalisme : politique et reconnaissance d'autrui (1994). 


\section{Québec : État et société. Tome I.}

Première partie. Le nationalisme québécois et la réforme constitutionnelle

\section{Chapitre 3 \\ “Le pluralisme et le dualisme.”}

Charles TAYLOR

[pp. 61-84.]

Y a-t-il des divergences à propos des valeurs entre les différentes régions du Canada ? Oui, mais en un sens, elles sont minimes. Il semble y avoir une similitude remarquable partout au pays, par-delà la différence entre francophones et anglophones, quand on parle des choses importantes de la vie. Même lorsqu'on traite des valeurs qui touchent précisément à la culture politique, on est généralement d'accord sur les questions de l'égalité, de la non-discrimination, de la primauté du droit, des mœurs de la démocratie représentative, à propos des mesures sociales, de la violence, des armes à feu et d'une foule d'autres sujets.

Il n'en a pas toujours été ainsi. Il y a cinquante ans, des différences marquées existaient entre les deux principaux groupes en matière de culture politique. Pierre Elliott Trudeau (1956) a écrit sur le sujet. Les atteintes à la primauté du droit sous Maurice Duplessis, même si on ne semble pas lui en avoir tenu rigueur - sa façon de traiter les témoins de Jéhovah et les communistes par exemple -, indiquent que le Québec et le Canada français avaient des idées différentes du Canada anglais sur la tolérance en matière de dissidence. Certaines personnes étaient disposées à croire que les deux sociétés attribuaient des valeurs bien différentes au maintien du consensus sur des vérités et des normes importantes lorsque celles-ci entraient 
en conflit avec la tolérance, la liberté ou la diversité admise. Non pas que le reste du Canada fût très libéral à cette époque. On y a fait la vie dure à diverses minorités et à bon nombre de dissidents. Cependant, les raisons précises de l'illibéralisme étaient remarquablement différentes au Québec, organisées, semble-t-il, autour des valeurs d'un catholicisme traditionnel et ultramontain. C'est pour ces raisons que les autres Canadiens se sont fait de la province une image exotique et alarmante.

Aujourd'hui, cette différence a disparu. En gros, on pourrait dire que le Canada français a rejoint le Canada anglais ; plus précisément, on pourrait dire que les forces internes du Québec qui ont toujours lutté pour une société libérale l'ont emporté. Il serait peut-être plus juste de dire que les deux parties du Canada ont été emportées dans le consensus libéral qui s'est établi dans le monde occidental à la suite de la Deuxième Guerre mondiale. Comme nous le verrons un peu plus loin, certains Canadiens anglais semblent ne pas croire à ce phénomène et entretenir un doute sur la présence des valeurs libérales au Québec, mais ce doute n'est pas fondé dans les années 1990. Ou s'il est fondé, c'est dans la même mesure que pour toute société des deux côtés de l'Atlantique, car aucune n'est exempte de racisme, de chauvinisme et d'autres maux de ce genre.

Ironiquement, au moment même où nous nous entendons sur ce point, la rupture est proche. Nous n'avons jamais été aussi près de la rupture dans notre histoire, bien que nos valeurs n'aient jamais été aussi uniformes. La voie de l'uniformité va au-delà du nivellement des différences entre deux grandes cultures. De plus, il $\mathrm{y}$ a eu une érosion graduelle des modèles urbains et ruraux si on considère les cinquante dernières années. L'influence extraordinaire des communications modernes a probablement aussi réduit les diverses différences régionales. 


\section{POURQUOI LE CANADA?}

Donc, quel est le problème ? Il survient lorsqu'on se pose un autre type de question appartenant aussi au domaine des valeurs au sens large. On ne se demande plus « À quoi les gens attachent-ils de l'importance ? » mais « À quoi sert un pays ? » En d'autres termes, « Autour de quelles bases d'unité devrait-on construire une entité politique souveraine ? » En un sens, cette question est étrange. Peu de pays se la poseraient. Néanmoins, elle survient ici parce qu'il y a des choix à faire et donc un besoin de justification. Ces choix existent pour nous - dans notre compréhension de la situation - même lorsqu'ils ne sont pas très plausibles, lorsqu'ils jouissent de peu d'appuis et ne font pas partie du jeu politique. Ils peuvent toujours s'offrir comme défi à l'autojustification puisqu'ils ont existé dans l'histoire, et nous retenons le principe que nos arrangements proviennent d'un choix qui excluait ces solutions.

Au Canada hors du Québec (CHQ) ${ }^{1}$, il y a deux solutions : le pays ou des parties du pays auraient pu s'unir, ou auraient pu se joindre aux États-Unis ; et les parties pourraient aussi avoir échoué dans leur union et, en réalité, elles pourraient un jour se désunir de nouveau. Il y a donc deux questions existentielles pour le CHQ : celle de l'unité et celle de la spécificité. Pour le Québec, il existe une grande question, trop bien connue et trop souvent à l'ordre du jour pour avoir besoin d'être décrite. Elle porte sur la décision de faire partie du Canada ou non et, dans l'affirmative, comment. Je tiens à préciser qu'aucune des solutions existentielles ne peut constituer un choix fort au CHQ de nos jours, mais cela ne les empêche pas de servir de points de référence à l'autodéfinition. À ce titre, ce sont des outils très utiles pour cerner la question : " Pourquoi existons-nous ? »

En un sens, les questions existentielles des deux sociétés sont interreliées. Peut-être que le CHQ n'aurait pas autant besoin de l'autodéfinition ni de répondre

Au Québec, nous parlons allègrement du Canada anglais, mais les personnes qui y vivent ne s'identifient pas à cette expression. Il nous faut un moyen pratique pour désigner le reste du pays comme entité, même s'il n'a pas d'expression politique pour le moment. Afin d'éviter d'utiliser l'expression au long, je prévois utiliser CHQ dorénavant dans ce document. J'espère que le lecteur ne considérera pas cette appellation comme un barbarisme usurpateur ou de l'égocentrisme québécois (bien qu'elle puisse refléter un peu des deux). 
à la question "À quoi sert le Canada ? », si le Québec n'avait pas l'intention de répondre à ses questions existentielles radicalement. Néanmoins, une fois que l'existence du pays a été menacée de la sorte, toutes les solutions réprimées remontent à la surface dans le CHQ aussi.

Quelles sont donc les réponses ? Il est plus facile d'exposer le problème en considérant d'abord le Canada anglais. La réponse y a toujours été évidente. Il y a longtemps, lorsque le Canada anglais portait le nom officiel d'« Amérique du Nord britannique ", la question de la spécificité ne se posait même pas et l'unité semblait le corollaire du besoin de spécificité face au géant américain. Toutefois, à mesure que disparaît l'aspect britannique, et même l'aspect anglais, du CHQ, cette affirmation est de moins [63] en moins valable comme réponse. Nous sommes tous des sujets de la reine, mais cela semble vouloir dire de moins en moins de choses à de plus en plus de personnes. Fait encore plus embarrassant, cela signifie encore beaucoup pour certaines personnes, mais absolument rien pour d'autres et ne peut donc pas servir de ferment pour l'unité.

Ce qui tient le Canada ensemble hors du Québec n'est donc plus une même origine et de moins en moins une même histoire. Cependant, on trouve les éléments de liaison dans les institutions politiques et les façons d'être. La coupure avec l'ancienne identité n'est pas totale, puisque l'aspect britannique se définit aussi largement sur le plan des institutions politiques : le régime parlementaire, une certaine tradition judiciaire et ainsi de suite. Le glissement s'est fait de façon continue, sans coupure brusque dans le passage de l'ancien au nouveau. Certains éléments se sont même perpétués, mais l'enrobage est différent.

Les Canadiens se sentent différents des Américains parce que a) ils vivent dans une société moins violente et moins écrasée par les conflits. Cette situation est en partie attribuable à la chance. Notre histoire n'a pas donné lieu à une guerre raciale latente dans les classes défavorisées, avec ses tensions continuellement entretenues dans les villes. La situation est aussi attribuable à la culture politique. Dès le début, les Américains ont accordé de la valeur à une défense des droits énergique et directe et, par conséquent, ils ne sont pas prêts à condamner la violence avec vigueur. On fait preuve de plus de tolérance envers la violence au sud de la frontière, on est plus disposé à l'accepter. C'est à cela qu'on peut attribuer le niveau de violence plus élevé aux États-Unis, de même qu'une série de penchants étranges de la société américaine, comme celui qui s'exprime dans la puissance du 
groupe de pression en faveur des armes à feu personnelles. Les Canadiens semblent accorder plus de valeur «à la paix, à l'ordre et au bon gouvernement ». C'est du moins notre façon de nous voir et il semble bien y avoir une certaine vérité dans cette perception qui est peut-être importante pour les buts que nous voulons atteindre.

Par conséquent, la population canadienne tolère plus facilement les règles et les restrictions justifiées par le besoin d'ordre. De plus, il existe un préjugé favorable (du moins au Canada anglais) qui offre le bénéfice du doute aux forces policières. Cela explique l'absence presque totale de protestations lors de la mise en vigueur de la Loi sur les mesures de guerre en 1970 ; cela explique aussi l'étrange hésitation de la population canadienne à condamner la GRC, même après la mise au jour de sa conduite douteuse.

On peut ajouter que la tolérance des Américains envers les conflits se prolonge également dans le domaine du droit. Ils sont plus procéduriers que nous le sommes. Ils croient que c'est une bonne chose, que cela paraît bien. Selon eux, personne ne devrait accepter d'idioties de la part des autres, alors que nous avons tendance à les déplorer. D'un point de vue américain, nous avons une soif insatiable de bêtise. Cependant, l'effet à long terme de la Charte de 1982 sera peut-être d'aplanir cette différence.

Les Canadiens se sentent différents des Américains aussi parce que b) ils considèrent que leur société politique est plus dévouée à l'aspect collectif, contrairement à la société américaine qui accorde plus d'importance à l'initiative personnelle. Des demandes visant à réduire la taille du gouvernement sont faites par des gens de droite dans les deux pays, mais leur idée sur ce que signifie réellement un appareil gouvernemental [64] allégé n'est pas du tout la même. Certes, il existe des différences régionales au Canada, mais en général les Canadiens sont fiers et satisfaits de leurs programmes sociaux, en particulier de l'assurance-maladie, et trouvent inquiétante leur absence aux États-Unis. Le fait qu'on a laissé croître la pauvreté et l'indigence dans les villes américaines au cours des années Reagan est normalement vu comme un aspect négatif de cette société. Les coutumes canadiennes ne sont peut-être pas aussi bonnes qu'on le croit, mais l'important est de considérer que la différence mérite d'être sauvegardée. 
Ainsi ces deux éléments, a) l'ordre public et b) la disposition collective, permettent de répondre à la question relative à la spécificité. Ils expliquent pourquoi nous sommes et voulons demeurer une unité politique distincte. Quelle est la réponse à la question de l'unité ? Pourquoi former un seul pays et quels buts communs devraient l'animer? En un sens, les arguments au point a) et au point b) peuvent aussi servir ici, si on pense (comme le font bon nombre de Canadiens instinctivement) que nous devons rester ensemble pour maintenir cette autre culture politique comme option viable en Amérique du Nord. Et puis le point b) peut être logiquement prolongé dans l'un des objectifs communs de la fédération canadienne au cours des dernières décennies, soit c) le partage équitable entre les régions des possibilités de réalisation individuelle et collective. Cette solidarité qui, à l'intérieur de chaque société, entraîne la création de programmes comme le régime d'assurance-maladie peut être perçue comme devant s'étendre logiquement à l'aide mutuelle entre régions.

Ainsi, le fédéralisme canadien a donné naissance aux pratiques de redistribution à grande échelle des ressources fiscales au moyen des paiements de péréquation et de la mise en marche des programmes de développement régional. Ces démarches contrastent aussi avec la pratique américaine récente et offrent une autre réponse à la question de la spécificité. Notre besoin d'égalité est probablement attribuable au fait que nous avons été mis face à des questions existentielles alors que nos voisins ne l'ont pas été depuis 1865. L'union fédérale canadienne a dû justifier sa propre existence à maintes reprises, et une plus grande solidarité régionale a pu découler de cette appréhension sous-jacente.

Mais ce principe de liaison a aussi été une source de division inquiétante, parce qu'il est grandement perçu comme un noyau d'aspirations démesurées et d'attentes déçues. Les principes de l'égalité régionale et de l'aide mutuelle s'opposent à ce qu'on perçoit comme la domination exercée par le Canada central sur les régions périphériques, caractérisée par un écart cruel entre les promesses et les réalisations. Il est devenu plus clair récemment que la déception prend deux formes plutôt différentes, ce qui reflète des idées variées de l'égalité régionale. En certains endroits, principalement dans les provinces de l'Atlantique, on déplore surtout que les programmes fédéraux n'aient pas réussi à améliorer les conditions économiques régionales. L'échec est lié à la question de l'aide mutuelle. Ailleurs, surtout dans l'Ouest du Canada, les gens se plaignent principalement du fait que 
leurs intérêts sont négligés : le Canada central dominant n'écoute pas les régions et passe outre à leurs demandes. L'échec est lié au problème du partage du pouvoir. Selon la première conception, le contrat implicite mais non respecté exige une redistribution aux régions plus pauvres. Selon l'autre conception, il exige un redressement des pouvoirs et de l'autorité en faveur des régions ayant moins d'influence aux points de vue démographique et économique. Dans un [65] cas, la promesse implicite est l'égalisation des revenus ; dans l'autre, elle porte sur le partage égal des pouvoirs entre les sociétés régionales.

Il est clair que la question de l'égalité régionale est très complexe au Canada, d'une part, parce qu'elle constitue un élément indispensable de la réponse à la question de l'unité et, d'autre part, parce que pour beaucoup de gens elle ne semble pas résolue. Par surcroît, nous sommes de moins en moins d'accord sur ce qu'elle signifie réellement.

Même si les choses allaient à merveille dans ce domaine, nous n'aurions toujours pas de réponse complète à la question de l'unité. Le Canada anglais devient au fil des ans de plus en plus diversifié et de moins en moins anglais. Le fait qu'il a toujours constitué une société d'immigrants, c'est-à-dire une société dont le fonctionnement est assuré par l'admission d'un flot régulier de nouveaux venus, et le fait qu'il ne peut plus espérer que les immigrants continuent d'être modelés selon son moule original, signifient que le Canada est de facto devenu multiculturel. Il ne peut espérer assimiler les nouveaux venus à un modèle préexistant puisque ce modèle est d'origine britannique, par conséquent ethnique. Aux États-Unis, où on a toujours eu la conviction d'être le lieu d'institutions politiques incomparables, la volonté de faire de tous des Américains pouvait se concrétiser rapidement. Au point de vue politique, l'identité canadienne n'a jamais été claire et, dans la mesure où elle a été rattachée à l'élément britannique, elle ne pouvait constituer la norme pour les nouveaux arrivants. D'abord parce qu'elle constituait uniquement l'identité d'une partie du pays et, ensuite, parce qu'elle ne pouvait être vue que comme un cadre ethnique parmi d'autres.

Pour les Canadiens, le Canada a moins l'allure d'un creuset que les États-Unis. Il y a une part de vérité dans cette affirmation. Comparativement à ce qui se passe dans la société voisine, les gens parlent ici d'une mosaïque canadienne. C'est même devenu pour certains une nouvelle facette de leur réponse à la question de la spécificité, sous la rubrique d) multiculturalisme. Cette appellation est loin d'être 
moins complexe. On se pose des questions au sein des deux cultures principales à propos du rythme et même des objectifs d'intégration ou d'assimilation des immigrants dans les sociétés anglophone ou francophone. Cette situation est particulièrement inquiétante au Québec, où on a beaucoup moins d'expérience historique dans l'assimilation des immigrants et où on trouve aussi une proportion beaucoup plus grande de la population qui est francophone pure laine.

Ainsi, la situation rend très urgente la définition d'une autre base pour l'unité, d'un point de référence commun sur l'identité, qui pourrait rallier des gens de divers cadres et régions. Fait étonnant, e) la Charte des droits en est venue à combler ce besoin au Canada anglais au cours des dernières années. Étonnant, parce qu'il y a neuf ans, elle n'existait pas. Il n'y avait pas davantage de mouvement de soutien pour demander son adoption avant qu'elle devienne une pomme de discorde entre les gouvernements fédéral et provinciaux dans la course au rapatriement de la constitution en 1981-1982. Mais le débat du lac Meech a démontré l'importance de la Charte au CHQ, pas seulement comme tremplin pour réclamer des droits additionnels mais aussi comme un élément important de cohésion entre tous les Canadiens. Pour beaucoup, la Charte en est venue, en l'espace de quelques années, à définir, en partie du moins, l'identité politique canadienne (Cairns, 1990a, p. 71-96 ; 1990b, p. 121-147). Aussi, [66] comme l'identité nationale au CHQ doit être définie pour ce qui est des institutions politiques pour les raisons données ci-dessus, l'événement a été décisif.

\section{POURQUOI LE QUÉBEC?}

Qu'en est-il du Québec ? Comment peut-il répondre à sa question existentielle ? La situation y est très différente. La question de la spécificité ne s'y pose pas. La langue et la culture suffisent à nous démarquer des Américains et aussi des autres Canadiens. Les arguments de a) à e) sont en grande partie perçus comme une bonne chose au Québec. En ce qui a trait au point a) - l'ordre public -, les gens ne se comparent pas beaucoup avec les États-Unis, mais il n'y a pas de doute que les Québécois sont spontanément du côté de l'ordre public et sont encore plus horrifiés par des conflits internes que les autres Canadiens. Le FLQ s'est subitement et totalement coupé de tout appui dès qu'il a assassiné Pierre Laporte. Les 
efforts des ex-membres du FLQ, faits avec plus ou moins de conviction, pour romancer leurs escapades lors du vingtième anniversaire de la crise d'Octobre, ne devraient pas porter à confusion à cet égard. La réaction au massacre des étudiantes de l'École polytechnique est aussi éloquente à ce titre. La société québécoise a bien plus réagi comme une famille blessée que comme une unité politique impersonnelle, à grande échelle.

En ce qui a trait au point b) - la disposition collective -, il va sans dire que les citoyens du Québec sont fiers de leurs programmes sociaux et tiennent à les conserver. Le point d) - le multiculturalisme - pose plus de problèmes. Comme politique fédérale, le multiculturalisme est parfois vu comme un moyen de refuser aux minorités francophones leur pleine reconnaissance, ou même de diminuer l'importance du fait français au Canada, le réduisant au statut d'une grande minorité ethnique. Même au Québec, la diversité grandissante de la collectivité francophone cause beaucoup d'animosité et d'inquiétude. Le point c) - l'égalité régionale et l'aide mutuelle - est généralement bien vu au Québec, et même la cinquième dimension e) - la Charte - était vue favorablement jusqu'à ce qu'elle soit perçue comme un instrument pour l'avancement des régimes d'uniformisation de la langue partout au Canada. Même de nos jours, les autres dispositions de la Charte sont très populaires.

Cependant, ces éléments n'apportent pas vraiment de réponse à la question : «À quoi sert un pays ? » Il existe une réponse évidente à cette interrogation qui a surgi sans cesse depuis plus de deux siècles : f) il faut un pays pour défendre ou promouvoir la nation. La nation dont on parle ici était à l'origine la nation canadienne-française. Depuis 1960, sans abandonner complètement la première formulation, on tend à l'appeler la nation québécoise. Cette nouvelle appellation ne dénote aucun changement dans l'identité ethnique, bien entendu. Elle reflète plutôt la notion, qui semble réaliste mais peut-être trop pessimiste, selon laquelle les vrais éléments de la nation canadienne-française appelés à survivre se trouvent uniquement au Québec.

Cependant, ce qui compte, c'est que l'argument au point f) fait de la survie ou de l'essor de la nation et de la langue un des premiers objectifs de la collectivité politique. Une entité politique ne méritera l'allégeance des gens que si elle contribue à réaliser [67] cet objectif. Le débat entre indépendantistes et fédéralistes au 
Québec donne simplement cours à divers jugements à propos du meilleur moyen d'y parvenir.

Si on ramène cela à la recherche d'une formule possible pour le Canada, cela signifie que, du point de vue du Québec, les points a) à e) sont peut-être attrayants, mais la solution choisie, pour que l'option canadienne soit jugée valable, doit contribuer à la survie et à l'épanouissement de la nation canadienne-française.

En pratique, cela suppose un certain dualisme. C'est à cette notion que faisaient référence les divers dirigeants du Québec lorsqu'ils décrivaient le Canada comme pacte entre deux nations ou deux peuples fondateurs.

Logiquement, ce dualisme devait exister à deux niveaux. Premièrement, i) cela signifiait que le français devait être reconnu comme langue au même titre que l'anglais dans la fédération. C'est-à-dire que le français devait avoir un statut distinct de celui d'une autre langue parlée par des immigrants, même s'il constituait l'idiome le plus important parmi les autres langues. Deuxièmement, ii) le dualisme impliquait que la nation canadienne-française, ou sa composante la plus nombreuse, devait avoir une certaine autonomie, une certaine capacité d'agir en tant qu'unité. Ces deux caractéristiques étaient en quelque sorte présentes dans le pacte original de la Confédération, mais dans le cas de i) - le bilinguisme - sous une forme partielle et mesquine. Le bilinguisme i) et l'autonomie du Québec ii) sont des exigences distinctes mais aussi liées. Il existe un certain degré de complémentarité dans la mesure où plus les droits linguistiques seront officiellement reconnus, moins le besoin d'autonomie politique se fera sentir. Ce qui est tragique au Canada, c'est que le point i) a peut-être été accordé trop tard et à contrecœur, et que cela a eu pour conséquence qu'on en est venu à formuler des demandes très exigeantes et irréversibles pour le point ii).

Ces deux exigences ont créé des problèmes. Le prolongement de i) au-delà de ses limites originales a soulevé une difficulté, puisque le CHQ, dans le cadre du développement de son multiculturalisme, a naturellement été forcé d'accorder à l'anglais le statut de langue commune et de séparer la langue de la culture. L'établissement de l'anglais comme langue principale ne supposait pas que les personnes de descendance anglaise avaient des privilèges ou étaient en quelque sorte supérieures. L'hégémonie de l'anglais devait être justifiée dans une perspective purement utilitariste. Dans cette structure, il était impossible de mettre l'anglais et 
le français sur un pied d'égalité. Hors du Québec, un statut distinct pour le français était rarement justifié par le nombre et certainement pas comme moyen de communication indispensable. Y accorder un tel statut paraissait du favoritisme injustifiable.

En deuxième lieu, les points i) et ii) se heurtaient à de la résistance en raison de la différence entre les valeurs du Québec et celles du reste du Canada. Cette différence dénote un préjugé de la part des protestants orangistes qui se répand dans beaucoup d'autres milieux en raison d'un soi-disant appel à des modes de pensée illibéraux au Québec. Cette différence a particulièrement joué contre ceux qui réclament davantage de concessions dans le domaine de l'autonomie politique.

Une des réalisations remarquables des trente dernières années, en particulier sous les gouvernements Trudeau, a été la mise en place d'un bilinguisme i) presque général. Cette démarche a provoqué une certaine animosité dans quelques secteurs et pourrait être décisive pour les négociations à venir. J'y reviendrai plus tard. Cependant, il n'y a [68] pas de doute qu'on a opéré un changement considérable. Quant à l'autonomie du Québec ii), de grands progrès ont également été réalisés. D'abord, la fédération canadienne s'est révélée un instrument flexible, pouvant donner beaucoup de pouvoir aux provinces. Ensuite, bien que les besoins du Québec aient été différents de ceux des autres provinces, un important statut distinct a été accordé de fait. Le Québec dispose de son propre régime de retraite, perçoit son impôt sur le revenu, possède un régime d'immigration distinct, etc.

C'est la reconnaissance officielle de l'autonomie du Québec qui a été bloquée. Pour donner au Québec l'autonomie dont il a besoin, sans déséquilibrer la fédération canadienne, il faudrait concevoir un type original de relations entre lui et les institutions et le gouvernement fédéraux. Bien qu'on y soit parvenu de facto dans une large mesure, il existe certaines réticences à lui accorder la reconnaissance de principe. C'est qu'il y a une profonde incompatibilité quant aux objectifs entre les deux parties du Canada. Bien que l'ancienne incompatibilité des valeurs semble avoir disparu, un nouveau conflit a surgi à propos des objectifs, des réponses à donner à la question «A quoi sert un pays ? »

Les exigences du point ii), concernant un statut distinct pour le Québec, s'opposent à celles sur l'égalité régionale c) telles qu'elles sont conçues par beaucoup de personnes au CHQ, et à une compréhension commune de la Charte e). Pour 
certains, le point c) inclut désormais l'égalité des provinces. La grande force morale du principe d'équité entre les régions a été mobilisée derrière la question juridique plutôt abstraite du statut constitutionnel respectif des provinces. Dorénavant, on semble faire fi de l'équité régionale si toutes les provinces ne sont pas placées sur le même pied. Un statut distinct peut être présenté comme une infraction à ce type d'égalité.

Fait encore plus grave, le statut distinct du Québec est clairement justifié en fonction de la défense et de la promotion de la nation canadienne-française $\mathrm{f}$ ). Cependant, c'est un objectif et, de nos jours, le nouveau patriotisme de la Charte a donné naissance à une philosophie des droits et de la non-discrimination qui doute fortement de la pertinence des objectifs collectifs. Elle peut les admettre uniquement s'ils sont clairement subordonnés aux droits individuels et aux dispositions relatives à la non-discrimination. Toutefois, pour ceux qui prennent ces objectifs au sérieux, cette subordination est inacceptable. La Charte et la promotion de la nation, comme on les comprend dans leurs limites respectives, sont placées sur la voie de l'affrontement. Les réactions à la loi 178 et à une bonne part du débat sur l'accord du lac Meech en disaient long à ce sujet.

Cette difficulté surgit quand il est question de l'autonomie du Québec ii), alors qu'elle avait été évitée à propos du bilinguisme i). Les dispositions en faveur du bilinguisme dans la législation fédérale peuvent être justifiées sur le plan des droits individuels. Elles garantissent que les francophones peuvent interagir avec les autres et obtenir des services gouvernementaux dans leur langue. Une fois que le français a, comme l'anglais, été ainsi reconnu, on considère que les droits individuels sont protégés. L'objectif collectif va encore plus loin cependant. Le but n'est pas seulement de voir à ce que les francophones soient servis en français, mais qu'il y ait encore des francophones à la prochaine génération : tel est l'objectif qui se dégage de l'argument énoncé au point f). Il ne peut être transposé en garanties de droits pour les francophones existants. En fait, en poursuivant cet objectif, on peut même se voir obligé de réduire leur [69] liberté de choisir, comme le fait la loi 101 au Québec, où les parents francophones doivent envoyer leurs enfants dans des écoles de langue française.

Conséquemment, les deux moitiés du Canada vont devoir s'affronter en raison de l'écart entre leurs réponses respectives à la question : "À quoi sert un pays ? » Plus précisément, il y a conflit entre le point c), l'égalité régionale, et le point e), 
la Charte, d'une part, et le point ii), l'autonomie du Québec, d'autre part. D'autres problèmes sont soulevés à propos du statut distinct. On se demande, entre autres choses, comment les Québécois vont pouvoir participer aux discussions quand le parlement fédéral va débattre des questions concernant les autres Canadiens, particulièrement quand ces questions divergeront grandement de celles touchant le Québec. Toutefois, je pense que cette difficulté est exagérée. Un très grand fossé doit se creuser entre les deux parties en cause pour que la situation fasse problème ${ }^{2}$.

\section{POURQUOI MAINTENANT?}

On pourrait se demander pourquoi l'affrontement doit avoir lieu maintenant. L'ancien Canada anglais, avant la législation sur le bilinguisme et la révolution Trudeau, était certainement plus fermé aux demandes du Québec. Il regimbait non seulement devant le point ii), l'autonomie du Québec, mais aussi devant le bilinguisme i). De plus, il s'immisçait beaucoup plus dans les affaires du Québec. À cette époque, la minorité anglaise, souvent appuyée par le gouvernement fédéral ou les institutions pancanadiennes comme le Canadian Pacific, fonctionnait uniquement en anglais, excluant, marginalisant ou rabaissant la langue française. Pourquoi les choses ne se sont-elles pas alors gâtées?

La réponse est que la séparation n'apparaissait pas comme une solution réaliste à cette époque, pour toutes sortes de raisons. Mentionnons d'abord une évaluation juste du rapport de force et une idée de ce que la majorité canadienneanglaise tolérait. Il y avait aussi un plus grand engagement envers les minorités francophones à l'extérieur de la province que de nos jours. Toutefois, le rôle éco-

2 Je ne tiens pas à m'attarder sur cette question dans ces pages, mais notre expérience et celle d'autres pays tendent à ébranler le fondement de notre inquiétude à ce sujet. Il ne faut pas oublier que le Québec a déjà un statut spécial. En 1964, les membres du Québec ont siégé à la Chambre des communes pendant le vote sur le Régime de pensions du Canada, à la suite d'une entente avec le Québec stipulant que la province aurait son régime. Le fait qu'il existe un régime distinct pour le Québec ne signifiait pas le désintéressement des Québécois en ce qui a trait aux arrangements canadiens. Au contraire, puisqu'en matière de transférabilité, chacune des parties s'intéressait grandement à l'autre. D'autres exemples de relations asymétriques de la partie au tout viennent à l'esprit, comme le gouvernement provincial qui a existé pendant de nombreuses années en Ulster. On a mis fin à l'expérience pour des raisons qui n'ont rien à voir avec la désuétude constitutionnelle. 
nomique restreint des Québécois francophones constituait un facteur important. Les Canadiens anglais jouaient encore un rôle prépondérant dans l'économie. Les grandes entreprises imposaient l'anglais; les anglophones occupaient les postes de direction et exerçaient plus que leur part de professions économiques-clés comme l'ingénierie. La situation causait de l'animosité sur bien des plans. En particulier, cette situation permit à la langue anglaise de s'attribuer dans la province une place qui n'était en aucune façon justifiée au point de vue démographique. À titre d'exemple, avant la révolution tranquille, à maintes et maintes reprises, les chefs syndicaux durent négocier en anglais avec les patrons, alors qu'ils représentaient une main-d'œuvre entièrement francophone. Ce déséquilibre contribua à établir un climat social où la société québécoise se sentait incomplète, dans lequel les fonctions essentielles étaient remplies par des agents venus de l'extérieur. La relation n'était pas aussi claire à cette époque, mais c'est ainsi qu'on pouvait éviter d'envisager la rupture totale. Avant 1960, la séparation n'était pas une option réaliste, même si l'abbé Groulx semble l'avoir considérée comme une possibilité à long terme.

Paradoxalement, au moment où on éliminait les animosités les plus grandes, où la marginalisation insultante et parfois menaçante de la langue française était en voie de s'effacer, à mesure que les Québécois francophones commençaient à prendre la place qui leur revenait dans l'économie, d'abord dans les secteurs public et parapublic (par exemple Hydro-Québec), puis dans le secteur privé, précisément à la suite de toutes ces réussites, la demande d'indépendance prit de l'ampleur. Elle en a pris jusqu'à devenir une des deux issues constitutionnelles d'importance, sur un pied d'égalité avec sa rivale fédéraliste et, depuis Meech, elle est même en avance sur elle. Tout cela pendant qu'à l'extérieur du Québec, au palier fédéral, le bilinguisme progresse et que les Québécois exercent plus de pouvoir que jamais auparavant. Ce sont les années du French Power. Certaines personnes de l'Ouest ont l'impression que le gouvernement fédéral est dirigé par les Québécois. Pourquoi la rupture s'annonce-t-elle à l'horizon ? Comment expliquer ce résultat paradoxal et même contradictoire ?

Une partie de la réponse, implicite dans les développements précédents, tient à ce que maintenant, pour la première fois, la solution semble concevable, possible, et même sûre. À cet égard, même la dernière décennie a été témoin d'un change- 
ment. En 1980, la plupart des Québécois considéraient encore la souveraineté comme une solution un peu effrayante. Les résultats du référendum ont clairement confirmé cette peur. En 1991, la situation ne semble plus la même. Une grande part de la différence serait attribuable au rôle de premier plan que jouent, semblet-il, les Québécois dans notre vie économique. Ce phénomène s'est étalé sur plusieurs décennies, mais comme c'est souvent le cas avec les perceptions publiques alimentées par les médias, on s'est soudainement rendu compte de la situation. Une grande vague de confiance a accompagné cette prise de conscience. Comme c'est souvent le cas avec les perceptions d'inspiration médiatique, nous passons facilement d'une exagération à l'autre. Les Québécois n'étaient pas aussi impuissants par le passé et ne sont pas aussi puissants aujourd'hui qu'ils le pensent. La séparation pourrait être beaucoup plus coûteuse économiquement qu'ils ne le croient présentement, et elle aurait été moins catastrophique que beaucoup le croyaient en 1980. Nous pourrions même assister à un autre revirement d'opinion au moment où la récession actuelle met les attentes en veilleuse. Toutefois, le changement fondamental est indéniable : la séparation est vraiment une solution concevable maintenant.

Pourtant, l'explication n'est pas complète. Prétendre le contraire serait dire que les Québécois n'ont jamais vraiment voulu autre chose, qu'ils ne faisaient qu'attendre le moment propice pour passer à l'action. Rien n'est plus faux. Beaucoup de liens rattachent les Québécois au Canada : d'abord, cette idée que la grande entité politique était le foyer de la nation canadienne-française, dont la présence s'étendait bien au-delà des frontières du Québec; puis un certain lien avec une patrie constitutionnelle qui était devenue familière et dont les dirigeants avaient contribué à la fondation. Pourtant, il n'y a jamais eu de patriotisme véritable envers le Canada. Ce type de sentiment était réservé à la nation canadienne-française. Il a récemment été transposé sur le Québec, comme segment viable de cette nation, mais à partir de là, il n'a jamais réussi à s'étendre à l'ensemble de l'unité politique.

C'est pourquoi les gens ont souvent comparé le Canada à un mariage de raison pour les Québécois. Cette comparaison est un peu en deçà de la vérité parce qu'elle ne [71] tient pas compte du lien multiforme avec le Canada. Toutefois il est vrai que, sur le plan émotionnel et dans un sens négatif, le patriotisme envers un Canada bilingue comptant deux nations ne s'est jamais développé. 
Cela ne suffit pas à expliquer la force de la solution indépendantiste de nos jours. Après tout, si le Canada est un mariage de raison, pourquoi l'abandonner lorsqu'il n'a jamais été aussi raisonnable, au moment où l'affaire semble plus favorable que jamais ? Certes, bon nombre de fédéralistes québécois défendent la cause du Canada aujourd'hui en utilisant précisément ces termes. Pourquoi n'ont-ils pas plus de succès ? Pourquoi ceux qui défendent cet argument sont-ils si ambivalents à son propos?

On peut très bien être induit en erreur sur cette question, parce que les opposants à ces partisans du fédéralisme rentable semblent vouloir les entraîner à leur suite et lutter pour prouver que la Confédération n'est pas avantageuse pour nous. En fait, la dynamique émotionnelle favorable à l'indépendance se trouve ailleurs. Cela tient beaucoup plus à une faillite sur le plan de la reconnaissance. Pendant des décennies, les dirigeants du Québec ont expliqué que la Confédération était un pacte entre deux peuples fondateurs, deux nations. Les choses n'ont jamais été vues ainsi à l'extérieur de la province. Cependant, l'affirmation des dirigeants ne visait pas surtout à donner le sens évident du pacte confédératif, en quelque sorte oublié par les autres - bien que ce soit souvent ainsi qu'on l'ait expliquée. Elle exprimait plutôt la conviction que c'est uniquement sous cette forme que la Confédération pouvait être acceptable pour les Canadiens français au bout du compte. Elle s'efforçait ainsi de toucher leur cœur et de respecter leur dignité.

En fait, dans la réalité, il fallait vivre avec des compromis dans lesquels le principe de la dualité avait un sens plutôt limité et était accepté à contrecœur. Il fallait travailler et vivre dans un pays qui, pour toutes sortes de raisons, était dirigé bien plus comme une nation où prévalait une culture dominante, avec plus ou moins de dispositions généreuses pour les minorités dans les régions. Les Canadiens d'aujourd'hui, dont certains peuvent encore vouloir se plaindre du nombre de langues présentes sur leur boite de céréales du matin, n'ont aucune idée de l'espace étroit qui était accordé au français aux plus sombres moments de notre histoire. En 1930, par exemple, les billets de banque étaient encore imprimés en anglais seulement.

Le Canada devait être accepté, mais jamais suffisamment pour lui donner son cœur, ou y trouver de la dignité. Il ne pouvait être accepté « dans l'honneur et l'enthousiasme », pour citer une expression qui est revenue si souvent au cours du drame du lac Meech. Sous l'acceptation rationnelle du mariage de raison, ce désa- 
veu a eu un effet profond. Il est facile de l'oublier parce que ceux dont le désir de reconnaissance est frustré ne veulent pas, bien entendu, présenter leur cause sous ce jour. C'est uniquement lorsqu'on obtient la reconnaissance qu'on consent à avouer à quel point on la désirait. Ainsi, l'expression « dans l'honneur et l'enthousiasme » est entendue lorsqu'il semble que les demandes ont finalement été satisfaites. Cependant, lorsqu'ils ne réussissent pas à obtenir la reconnaissance, les opposants au Canada fédéral prétendent que personne n'a jamais voulu de symboles, que le choix de l'indépendance se fait sur les plans réalistes du pouvoir et de la prospérité, que l'attitude du partenaire canadien-anglais ne signifie rien pour eux. Dans toute cette discussion, ils protestent un peu trop.

La force actuelle de l'indépendantisme est donc en partie attribuable à la nouvelle assurance des Québécois, et en partie au fait que le Canada n'a jamais constitué une [72] nation pour eux. Pour une grande part, elle est attribuable au fait qu'ailleurs on a toujours refusé de comprendre le Canada d'après les seuls paramètres qui auraient pu leur permettre de l'accepter pleinement. Ces paramètres sont précisés, entre autres, dans la conception dualiste du pays. Certes, cette vision était inacceptable telle quelle pour le reste du pays, qui ne se voyait pas lui-même comme une «nation ». De ce point de vue, les Canadiens français ont tenté d'imposer une identité symétrique à leurs partenaires. Cette tentative n'a pas encore été totalement abandonnée, comme l'indiquent les discussions qui alimentent le Québec de nos jours. J'y reviendrai. Néanmoins, on pouvait distinguer une exigence fondamentale dans cette présomption à l'égard de l'autre. Cette exigence portait sur la reconnaissance de la nation canadienne-française comme composante essentielle du pays, comme entité dont la survie et l'essor constituent des enjeux majeurs pour le Canada en tant que société politique. Si une telle exigence avait été acceptée, peu eût importé comment le reste du pays se définissait.

En réalité, le pays a fini par résoudre assez bien ce dilemme par l'instauration du bilinguisme fédéral, grâce aux progrès réalisés par certaines minorités francophones ailleurs au moyen d'un statut administratif distinct de facto pour le Québec. Toutefois, il manquait encore la reconnaissance officielle que cette démarche faisait partie de notre objectif en tant que fédération. C'est ce qui explique l'importance de l'accord du lac Meech et pourquoi son échec aura des conséquences si tragiques. Si on prend la peine d'écouter ce que les gens disent au Québec, la situation peut sembler étrange. Bon nombre de Québécois n'ont jamais même admis 
être en faveur de Meech ou ont exprimé un soutien mitigé. Fondamentalement, tous les indépendantistes ont choisi cette voie. Ceux qui doutaient du Canada anglais couvraient leurs arrières, ne voulant jamais avouer que la reconnaissance comptait pour eux. Néanmoins, leur réaction si énergique à l'échec de Meech montre à quel point cette façade était trompeuse.

Meech était important parce que, pour la première fois, la reconnaissance de la dualité canadienne et le rôle distinct du Québec étaient inscrits dans un énoncé portant sur l'essence même du Canada. Le fait que l'accord n'accordait aucun pouvoir additionnel au Québec a grandement diminué son importance en ce qui a trait précisément à cette déclaration d'intention. Après toutes ces années de nonreconnaissance, et dans le contexte d'un mariage de raison qui n'a pas permis de gagner les cœurs ni de rehausser le sentiment de dignité, on peut facilement s'expliquer l'importance de cette déclaration. On peut aussi la comprendre par le regard de la génération actuelle qui ne possède pas la timidité de ses ancêtres face à une rupture possible et qui constate avec un peu de surprise, et même parfois avec mépris, que ses prédécesseurs ont accepté cette non-reconnaissance pendant si longtemps. Le fait de subir un refus (même si l'accord ne constituait qu'une déclaration d'intention) prend une importance décisive. Voilà qui explique l'énoncé si souvent répété que Meech contenait nos conditions minimales.

Avec l'échec de Meech, un déclic s'est fait, et il est facile à définir. Les Québécois ne veulent plus vivre dans une structure qui ne reconnaît pas pleinement leurs objectifs nationaux. Au début des années 1980, après la défaite du « oui » au référendum, beaucoup ont joué avec l'idée d'accepter le mariage de raison et d'en faire une réussite, avec ou sans reconnaissance. La nouvelle confiance était peutêtre aussi motivée par cette position différente qui marginalisait la question de la reconnaissance. Après tout, si on connaît sa valeur, pourquoi a-t-on besoin des autres ? Mais en un sens, Meech [73] supprimait cette possibilité, justement parce qu'il ranimait l'espoir de la reconnaissance. Dorénavant, nous sommes irrémédiablement engagés sur une autre voie. (Non pas que je pense que la voie raisonnable aurait pu durer très longtemps de toute façon ; elle était toujours à la merci d'un fait nouveau.)

Il reste à expliquer l'euphorie extraordinaire notée par tous les observateurs dans les foules qui ont célébré la fête nationale du Québec, la Saint-Jean, en 1990. Pourquoi les Québécois se sentaient-ils si unis et si soulagés, comme si l'échec de 
Meech leur avait enlevé un poids de la conscience ? Je pense que cette réaction est attribuable au fait que la longue division et la longue hésitation entre le consentement raisonnable à une structure qui ne les reconnaissait pas et l'insistance pour voir leurs objectifs nationaux acceptés ouvertement étaient enfin résolues. La situation polarisait les Québécois et ce, de façon plutôt pénible au moment du référendum de 1980 qui causa des tensions profondes dans nombre de familles. En plus, les Québécois étaient aussi divisés en eux-mêmes. Enfin, le long conflit, la longue hésitation, la grande ambivalence prenaient fin. Les Québécois savaient ce qu'ils voulaient de toute structure politique à venir sur la moitié nord du continent. On avait retrouvé le consensus, mais aussi une forme d'unité psychique. Un certain type de compromis semblait révolu à jamais.

Qu'est-ce que cela signifie pour l'avenir ? Cela signifie que l'exigence ii) pour l'autonomie du Québec est devenue urgente et pratiquement non négociable. Cette situation entraîne un danger réel de rupture entre les deux parties du pays. Ainsi, il s'ensuit que les Québécois n'accepteront pas une structure dans laquelle leurs aspirations collectives ne seront pas complètement et ouvertement reconnues. Ce refus est déjà apparent dans l'exigence que les négociations se tiennent à deux entre Québec et Ottawa. On croit que, de cette façon, le statut du Québec en tant que société distincte est reconnu. Tout cela se produit au moment où le patriotisme tout neuf du CHQ autour de la Charte le rend incapable de reconnaître la légitimité des objectifs collectifs et où l'aliénation régionale accorde une plus grande force au principe de l'égalité des provinces. Le terrain d'entente semble se rétrécir rapidement.

\section{DROITS ET NATIONS}

Ces demandes peuvent-elles être conciliées ? Examinons les différents conflits l'un après l'autre, à commencer par celui qui existe entre la Charte et les objectifs collectifs du Québec. Notre Charte respecte la tendance de la dernière moitié du $\mathrm{XX}^{\mathrm{e}}$ siècle et offre une base pour le contrôle judiciaire sur deux points fondamentaux. Premièrement, elle protège les droits de la personne de diverses façons. Deuxièmement, elle garantit le traitement équitable des citoyens sous divers as-

pects ; autrement dit, elle met les gens à l'abri de la discrimination fondée sur un 
certain nombre de motifs sans fondement, comme la race ou le sexe. Il y a bien d'autres choses dans notre Charte, y compris des dispositions sur les droits linguistiques et les droits des autochtones, qui pourraient être comprises comme l'attribution de pouvoirs aux collectivités, mais les deux thèmes que j'ai nommés dominent dans l'esprit des gens.

Cela n'est pas un hasard. Ces deux types de dispositions sont très courants dans les programmes de droits implantés de longue date qui constituent la base du contrôle judiciaire. En ce sens, le monde occidental, peut-être même le monde entier, suit le précédent américain. Les Américains ont été les premiers à écrire et à enchâsser une déclaration des droits pendant le processus de ratification de leur constitution et à l'imposer comme condition à son éventuelle acceptation. On pourrait dire qu'ils n'étaient pas vraiment précis au sujet de la récupération judiciaire comme méthode pour protéger ces droits, mais cela est rapidement devenu la coutume. Les premières modifications protégeaient les personnes, et parfois même les gouvernements des États ${ }^{3}$, contre l'empiétement par le nouveau gouvernement fédéral. C'est après la guerre civile, au cours de la période de reconstruction triomphale, et en particulier avec le quatorzième amendement, qui faisait appel à une protection équitable pour tous les citoyens par les lois, que le thème de la non-discrimination est devenu le centre du contrôle judiciaire. Il est maintenant à égalité avec l'ancienne norme de la défense du droit individuel et, dans la conscience populaire, peut-être même prépondérant.

Il faut noter que, pour un bon nombre de personnes au Canada anglais, une société politique qui adopte certains objectifs collectifs menace d'aller à l'encontre des dispositions fondamentales de notre Charte ou même de toute déclaration des droits. En premier lieu, les objectifs collectifs peuvent exiger des restrictions relatives à la conduite des personnes, restrictions qui pourraient violer leurs droits. Pour beaucoup de Canadiens non francophones, au Québec et hors du Québec, ce

3 Par exemple, à l'origine, le premier amendement, qui interdit au Congrès d'établir une religion, n'était pas destiné à séparer l'État et l'Église. Il a été mis en vigueur à un moment où beaucoup d'États avaient mis en place des Églises et il avait simplement pour but d'empêcher le nouveau gouvernement fédéral d'intervenir ou d'annuler ces arrangements locaux. C'est uniquement plus tard, après le quatorzième amendement, à la suite de la doctrine dite de «l'incorporation », que ces restrictions envers le gouvernement fédéral ont été étendues à tous les gouvernements, peu importe à quel niveau ils se trouvent. 
résultat tant appréhendé s'était déjà concrétisé avec la législation linguistique du Québec. Par exemple, la loi 101 régit le type d'école dans laquelle les parents doivent envoyer leurs enfants et, dans l'exemple le plus connu, elle prohibe certains types d'affichage commercial. La Cour suprême a jugé cette disposition contraire à la Déclaration des droits du Québec de même qu'à la Charte, et elle a été remise en vigueur parce qu'on a invoqué la clause dérogatoire (article 33 de la Loi constitutionnelle, 1982).

En deuxième lieu, même si ce n'était pas le cas, on peut croire que le fait d'adopter des objectifs collectifs au nom d'un groupe national est discriminatoire en soi. Dans le monde moderne, les citoyens d'une certaine entité politique n'appartiendront pas tous au groupe national favorisé, et il en sera toujours ainsi. On peut penser que ce phénomène en soi entraîne une certaine discrimination. Si on fait abstraction de cette opinion, la poursuite de la fin collective va, selon toute probabilité, requérir un traitement différent pour les personnes vivant à l'intérieur de cette société par rapport aux personnes de l'extérieur. Ainsi, la clause sur l'éducation de la loi 101 interdit (en gros) aux francophones et aux immigrants d'envoyer leurs enfants dans des écoles de langue anglaise, mais elle permet aux Canadiens anglophones de le faire.

Cette idée que la Charte va à l'encontre de la politique fondamentale du Québec était une des principales raisons de l'opposition du CHQ à l'accord du lac Meech. L'inquiétude portait sur la clause de la société distincte, et l'exigence de modification commune insistait pour que la Charte soit protégée contre cette clause, ou ait la préséance sur celle-ci. Cette demande découlait sans aucun doute d'un certain préjugé anti-Québec de source ancienne, vestige de l'image d'un «Québec écrasé par les soutanes ». Ainsi, divers groupes de femmes ont exprimé la crainte que les gouvernements du Québec, pour faire augmenter le taux de natalité, adoptent une politique de type [75] Ceaucescu, en interdisant l'avortement et en rendant la contraception plus difficile. Cependant, même en excluant la sottise, le mépris et la mauvaise volonté, il demeure qu'il y a deux types d'arguments à considérer sérieusement. D'abord, il existe une différence philosophique réelle dans les fondements de la société libérale. Ensuite, il y a une divergence d'opinions en ce qui a trait au fondement de l'unité nationale.

Penchons-nous d'abord sur la différence philosophique. Ceux qui considèrent que les droits individuels doivent toujours passer en premier et, avec les clauses 
de non-discrimination, doivent avoir préséance sur les objectifs collectifs se basent souvent sur un modèle libéral qui s'est répandu progressivement dans le monde anglo-américain. Bien entendu, les États-Unis constituent sa source et il a récemment été élaboré et défendu par certaines des grandes sommités, philosophes et experts en droit, appartenant à ce modèle comme John Rawls (1971, 1985), Ronald Dworkin (1977, 1978), Bruce Ackerman (1980) et d'autres. L'idée maitresse se présente sous plusieurs formes, mais celle qui nous touche de plus près a été exposée par Dworkin (1978) dans un texte succinct intitulé «Liberalism ».

Dworkin établit une distinction entre deux types d'engagement moral. Nous avons tous une opinion quant aux buts de la vie, à ce qui constitue la qualité de la vie, et à ce que nous et les autres devons tenter d'obtenir. Nous reconnaissons aussi qu'il existe une obligation d'interagir avec justice et équité les uns avec les autres, peu importe comment nous concevons nos buts. Nous pourrions appeler ce type d'engagements procéduraux, et ceux qui touchent les buts de la vie, des engagements substantifs. Dworkin déclare qu'une société libérale est celle qui, en tant que société, n'adopte aucune vision substantive sur les buts de la vie. Cette forme de société est plutôt unie autour d'engagements procéduraux solides, vouée à traiter les personnes avec un même respect. La raison pour laquelle le régime comme tel ne peut adopter de vision substantive, ne peut permettre par exemple que l'un des objectifs de la législation soit de rendre les gens vertueux d'une façon quelconque, c'est que cela enfreindrait sa norme procédurale. Étant donné la diversité des sociétés modernes, certaines personnes à l'exclusion d'autres seraient immanquablement engagées envers leur conception de la vertu. Elles pourraient être en majorité ; en fait, il est probable qu'elles le seraient, car autrement, une société démocratique n'adopterait pas leur point de vue. Néanmoins, cette vision ne serait pas partagée par tous et, en adoptant cette perspective substantive, la société ne traiterait pas la minorité dissidente avec un même respect. Elle dirait en fait à ses membres : "Votre opinion n'est pas aussi valable, aux yeux de ce régime, que celle de vos compatriotes plus nombreux. »

De grandes hypothèses philosophiques sous-tendent cette vision du libéralisme, qui est influencée par la pensée d'un philosophe allemand de la fin du XVIII ${ }^{\mathrm{e}}$ siècle, Emmanuel Kant. Entre autres caractéristiques, cette vision considère que la dignité humaine est constituée en grande partie par l'autonomie, c'est-à-dire l'apti- 
tude de chaque personne à déterminer pour elle-même une vision de l'êthos et de l'excellence qui lui convienne. Cela revient à dire que la dignité ne relève pas en priorité d'une compréhension quelconque de la vie bien vécue, de sorte que le fait pour une personne d'abandonner cette vie signifierait porter atteinte à sa propre dignité ; elle est davantage reliée au pouvoir d'étudier et d'adopter pour soi une vision quelconque. Nous ne respectons pas ce pouvoir également chez tous les sujets, dit-on, si nous élevons le résultat [76] des délibérations de certains individus au-dessus de celui d'autres personnes. Une société libérale doit demeurer neutre en ce qui a trait à la valeur des modèles et des objectifs de vie et s'en tenir à s'assurer que, peu importe leur façon de voir les choses, les citoyens interagissent de façon juste entre eux et que l'État agit avec équité envers tous.

La popularité de cette vision de l'agent humain, perçu avant tout comme maître de ses choix en matière d'autodétermination ou d'auto-expression, permet d'expliquer pourquoi ce modèle de libéralisme est si puissant. Cependant, il faut aussi tenir compte du fait qu'il a été recommandé avec force et intelligence aux États-Unis, précisément dans le contexte des doctrines constitutionnelles de contrôle judiciaire ${ }^{4}$. Il n'est donc pas surprenant qu'on accepte finalement l'idée, abstraction faite de ceux qui pourraient souscrire à une philosophie précise dérivée de celle de Kant, qu'une société libérale ne peut accepter l'imposition publique d'une conception du bien. Selon Michael Sandel (1984), cette conception dite de la république procédurale exerce une très forte influence sur le débat politique aux États-Unis, et elle a permis de placer de plus en plus l'accent sur le contrôle judiciaire à partir des textes constitutionnels, aux dépens du processus politique courant qui consiste à regrouper des majorités dans l'intention d'entreprendre des démarches législatives.

Toutefois, une société ayant des objectifs collectifs comme le Québec ne respecte pas ce modèle. Il est fondamental pour les gouvernements du Québec que la survie et l'épanouissement de la culture française au Québec constituent un bien. La société politique n'est pas impartiale envers ceux qui accordent de la valeur au fait de rester fidèle à la culture de nos ancêtres et envers ceux qui peuvent vouloir s'en soustraire au nom d'un objectif individuel quelconque d'épanouissement de soi. Après tout une société libérale procéduraliste pourrait adopter un objectif

4 Voir, par exemple, les arguments fournis par Lawrence Tribe dans Abortion : The Clash of Absolutes, New York, Norton, 1990. 
comme la survie. On pourrait considérer la langue française, par exemple, comme une ressource collective dont chacun peut vouloir user et que chacun peut vouloir préserver, comme on le fait pour l'air pur ou les espaces verts. Cependant, cette façon de voir les choses n'englobe pas la totalité de l'influence des politiques conçues pour la survie culturelle. Il ne suffit pas que la langue française puisse être utilisée par ceux qui le désirent. Cela peut sembler avoir été l'objectif de certains aspects de la politique fédérale de bilinguisme au cours des vingt dernières années. Il faut aussi s'assurer qu'il y aura une collectivité de gens ici dans l'avenir qui voudront profiter de cette possibilité. Les politiques axées sur la survie cherchent activement à faire en sorte que le nombre de membres de la collectivité touchée se maintienne ou augmente, par exemple en veillant à ce que les générations montantes continuent de s'identifier comme francophones. Impossible de considérer qu'elles ne font qu'offrir une option aux personnes de l'époque contemporaine (Kymlicka, 1989) ${ }^{5}$.

Par conséquent, les Québécois, et ceux qui accordent la même importance à ce type d'objectif collectif, ont tendance à opter pour un modèle plutôt différent de société libérale. À cet égard, une société peut être organisée en fonction d'une définition de ce qu'est le bien dans la vie sans que cela constitue une dévalorisation pour ceux qui ne partagent pas personnellement cette définition. Ainsi, la nature du bien exige qu'on cherche à l'obtenir en groupe, et c'est pourquoi il constitue un objectif politique. Selon cette conception, une société libérale se distingue par sa façon de traiter les minorités, [77] y compris celles qui ne partagent pas la définition publique du bien, et avant tout par les droits qu'elle accorde à tous ses membres. Toutefois, dans ce cas, les droits en question sont perçus comme les droits fondamentaux et essentiels qui ont été reconnus comme tels à l'origine de la

5 Selon Kymlicka (1989), ce que j'ai appelé le libéralisme de procédure peut être rendu compatible avec la défense des droits collectifs et la survie culturelle dans certains cas. Kymlicka, contrairement à la plupart des auteurs américains, écrit avec une grande connaissance de la situation canadienne et s'engage pleinement dans la défense des droits des autochtones du pays. En souscrivant à une politique "d'intérêt moral neutre », c'est-àdire à une conception de l'État libéral comme étant neutre vis-à-vis des définitions de ce qu'est le bien dans la vie (p. 76), il estime néanmoins que les droits culturels collectifs peuvent être défendus en s'appuyant sur le fait que les membres de certaines collectivités menacées seraient privés des conditions de décisions intelligentes et autogénérées à propos de la nature du bien si les « structures culturelles » par l'entremise desquelles elles peuvent obtenir cette possibilité étaient minées (p. 165). Si le raisonnement de Kymlicka l'emportait, il comblerait le fossé entre les deux modèles de libéralisme que je compare dans ce chapitre. 
tradition libérale : les droits à la vie, à la liberté, à un jugement équitable, à la liberté d'expression, à la pratique libre d'une religion, etc. Selon ce modèle, on exagère un peu, on néglige dangereusement une limite importante lorsqu'on parle de droits fondamentaux pour des choses comme l'affichage commercial dans la langue de son choix. Il faut faire une distinction entre, d'une part, les libertés fondamentales, celles sur lesquelles il ne faudrait en aucun cas empiéter et qui donc devraient être ancrées solidement dans la tradition, et, d'autre part, les immunités et privilèges qui sont importants mais qui peuvent être révoqués et restreints pour des raisons de politique publique - bien qu'une excellente justification soit nécessaire dans ce cas.

Une société ayant de grands objectifs collectifs peut être libérale, à cet égard, à condition de respecter la diversité, en particulier en ce qui a trait aux personnes qui ne partagent pas ses objectifs et à condition d'offrir une protection adéquate des droits fondamentaux. Il y aura certes des tensions et des difficultés dans la poursuite collective de ces buts, mais ceux-ci ne sont pas incompatibles et les problèmes ne sont pas plus grands que ceux auxquels doit faire face toute société libérale, appelée par exemple à combiner la liberté et l'égalité ou la prospérité et la justice.

Voilà deux visions incompatibles de la société libérale. Une des grandes sources de notre désaccord vient de ce qu'elles en sont arrivées à s'affronter directement au cours des dix dernières années. La résistance à l'idée de la société distincte qui exigeait qu'on donne préséance à la Charte est née en partie de la propagation de la perspective procédurale au Canada anglais. De ce point de vue, en attribuant l'obligation de promouvoir la société distincte du Québec à un gouvernement, on reconnaissait un objectif collectif. Il fallait neutraliser ce mouvement en le subordonnant à la Charte préexistante. Dans la perspective québécoise, cette tentative visant à imposer un modèle procédural de libéralisme ne ferait pas que priver la clause de société distincte d'une partie de sa force comme règle d'interprétation, elle laisserait aussi présager le rejet du modèle de libéralisme sur lequel s'est peu à peu fondée cette société. Il y a eu beaucoup de perceptions erronées dans les deux sociétés au cours du débat du lac Meech, comme je l'ai mentionné ci-dessus. Cependant, chaque société y a deviné quelque chose de vrai à propos de l'autre - et n'a pas aimé ce qu'elle a vu. Le CHQ a senti que la clause de la « société distincte » rendait légitimes les objectifs collectifs. Le Québec a vu que la dé- 
marche pour donner préséance à la Charte imposait une forme de société libérale étrangère à laquelle le Québec ne pourrait jamais se conformer sans renoncer à son identité. Dans ce contexte, les protestations des patriotes de la Charte disant qu'ils n'étaient pas « contre le Québec » sonnaient faux.

Voilà une des grandes sources de désaccord. Il y en a une seconde, reliée à la première. La Charte a acquis une importance considérable au CHQ, non seulement en raison de la force grandissante du libéralisme de procédure, mais aussi parce que dans la diversité croissante de cette société multiculturelle, on est à la recherche de nouvelles bases d'unité. Le CHQ a aussi trouvé sa raison d'être en partie dans ses institutions politiques, pour les motifs énumérés plus haut. Bien que la Charte offre un semblant de [78] réponse à la question de la spécificité parce qu'elle nous fait ressembler davantage aux États-Unis, elle peut néanmoins donner une réponse satisfaisante à la question de l'unité. Les deux motifs du patriotisme de la Charte se recoupent ici. À mesure que le pays se diversifie, nous sommes de plus en plus conscients des divergences dans nos conceptions de la nature du bien. Il en découle donc que ce qui pourrait et devrait nous rassembler, ce sont effectivement les normes "procédurales » qui régissent nos rapports sociaux. Le libéralisme de procédure non seulement commence à paraître de plus en plus plausible en lui-même, mais il semble aussi être le seul terrain d'entente incontestable.

Toutefois, si la Charte sert réellement de terrain d'entente, il devient alors difficile d'accepter que sa signification et sa portée puissent être modulées dans une partie du pays par un élément comme la clause de la société distincte et s'y appliquer de façon autre que dans les autres régions. La résistance opposée à cette clause de l'accord du lac Meech est venue en partie du fait qu'on croit que la Charte, à tout le moins, doit s'appliquer à tous les Canadiens de la même manière. Si le lien procédural est la seule chose qui peut nous garder ensemble, sans causer de discrimination, il ne doit souffrir aucune exception.

Ce conflit peut-il être résolu ? En un sens, non. Une partie du pays tente de le garder uni autour d'un modèle de libéralisme que l'autre partie ne peut accepter. S'il doit y avoir consensus, la première partie doit céder. En un autre sens, le terrain d'entente possible est facile à déterminer. Les libéraux procéduraux du Canada anglais n'ont qu'à reconnaître d'abord qu'il existe d'autres modèles possibles de société libérale, puisque leurs compatriotes francophones veulent vivre selon une 
de ces solutions de rechange. Il devient évident que le premier élément de cette affirmation est vrai lorsqu'on regarde la vaste gamme de sociétés libres du monde contemporain, en Europe et ailleurs, plutôt que de lorgner uniquement du côté des États-Unis. L'exactitude du second élément devrait être évidente pour quiconque possède une connaissance minimale de l'histoire et de la politique du Québec.

Néanmoins, une fois qu'on a accepté les deux éléments, il devient clair que le fait de placer le libéralisme de procédure à la base de l'unité canadienne n'est pas justifié et ne peut qu'échouer. La raison en est que cette façon de faire impose le modèle d'une société à une autre et, dans la situation de la démocratie canadienne à la fin du $\mathrm{XX}^{\mathrm{e}}$ siècle, cela ne peut réussir. Notre seule façon de pouvoir coexister est d'accepter d'être en désaccord sur ce point. Cela signifie-t-il pour autant que nous puissions coexister uniquement sous la forme de deux sociétés indépendantes, peut-être liées de manière assez souple par des institutions supranationales ? Voilà la thèse des souverainistes du Québec. Pourtant, cette thèse ne m'a jamais semblé aller de soi. Elle s'impose uniquement dans la mesure où les libéraux procéduraux ne démordent pas du principe qu'ils ne peuvent accepter de partager le pays avec des gens qui vivent selon un autre modèle. Une rigidité de ce genre est apparue au cours du débat du lac Meech. Si c'est là le dernier mot du CHQ, alors certes les indépendantistes ont raison et il n'y a pas d'autre solution que la souveraineté-association.

[79]

\section{ÉGALITÉ DE QUOI ?}

Le deuxième grand domaine de désaccord se situe entre les demandes visant le statut distinct pour le Québec et les exigences d'une égalité régionale, si on interprète celle-ci comme étant l'égalité entre les provinces. Toutefois, alors que pour les deux modèles de libéralisme il y a une différence philosophique réelle qui sous-tend toute l'opposition, il demeure encore beaucoup de perceptions erronées et de malentendus à propos de l'égalité.

En fait, les deux exigences proviennent de programmes bien différents, comme on l'a souvent noté. L'exigence d'un statut distinct a normalement comme ob- 
jectif de permettre au Québec d'assumer une plus vaste gamme de responsabilités et donc d'obtenir une plus grande autonomie. L'appel à l'égalité régionale provient généralement de personnes qui considèrent que leurs intérêts n'ont pas reçu toute l'attention nécessaire dans l'élaboration de politiques fédérales et qui désirent donc être davantage écoutées à ce chapitre. D'un côté, on veut se distancier un peu plus du gouvernement central et du Parlement; de l'autre, on veut plus de pouvoir au sein de ces mêmes institutions. Voilà pourquoi, au cours des dernières années, ces demandes ont pris la forme d'un appel à la modification des institutions fédérales, notamment du Sénat.

Vues sous cet angle, ces deux exigences ne sont pas logiquement opposées. Certes, elles peuvent se nuire sous bon nombre d'aspects. Les provinces qui souhaitent un gouvernement fédéral plus actif afin d'égaliser les conditions dans les régions ont peur que le fait d'accorder de trop grandes compétences au Québec diminue le pouvoir d'agir du centre. Ce phénomène pourrait bien se produire, mais pas obligatoirement. Ce conflit n'apparaît pas aussi logique que celui qui existe entre l'égalité de toutes les provinces, d'une part, et la cession de pouvoirs spéciaux à l'une d'entre elles, d'autre part. Il est possible de rendre compatibles les exigences d'un statut distinct et celles d'un gouvernement central fort. Ce qui a rendu cela difficile en pratique est précisément le refus d'abandonner l'uniformité. Cela signifie qu'une concession quelconque au Québec devait être offerte aux autres provinces. Heureusement, chaque concession n'a pas toujours été réclamée, de sorte que le Québec a fini par obtenir un statut distinct de fait, comme je l'ai noté précédemment. Cependant, il n'a jamais été possible de progresser dans cette direction ouvertement et explicitement, étant donné la pression exercée par le désir d'uniformité. Dans l'accord du lac Meech, conçu pour résoudre les problèmes du Québec, la plupart des points accordés au Québec ont dû l'être aussi à toutes les autres provinces.

Le vocabulaire de l'égalité entre les provinces est en fait une source de confusion, masquant la réalité des enjeux et rendant la solution plus difficile. La difficulté d'application du concept d'égalité est reconnue et dépend de l'aspect que l'on choisit de privilégier. On pourrait dire que le Québec doit avoir des pouvoirs dont les autres n'ont pas besoin pour venir à bout de problèmes et pour assumer une vocation que les autres provinces n'ont pas. Par conséquent, cet aspect pourrait être vu comme un pas vers l'égalité (à chaque province selon ses tâches), et non le 
contraire. De plus, le statut distinct n'a rien à voir avec le fait de placer plus de force au centre. Il suppose quelque chose de bien différent.

Tout cela devrait nous encourager à penser qu'il n'est peut-être pas au-dessus de l'intelligence humaine de découvrir une façon de satisfaire ces diverses exigences. Il y a a) des provinces qui veulent une plus grande participation aux décisions du gouvernement fédéral. Il y en a d'autres qui, bien que n'étant pas indifférentes au premier objectif cité, se préoccupent principalement de b) maintenir un gouvernement fédéral fort et actif à rechercher l'égalisation économique et sociale entre les régions. Puis il y a le Québec qui c) veut les pouvoirs qu'il croit essentiels à la conservation et à la promotion de sa société distincte.

À cela, il faut maintenant ajouter les revendications autochtones. Cela signifie que nos arrangements doivent répondre au besoin d'autonomie politique et d'autogestion des diverses nations. En pratique, cela peut signifier qu'il faudra prévoir une nouvelle forme de compétence au Canada, qui sera peut-être plus faible que celle des provinces. Toutefois, cette structure politique différera des municipalités, qui constituent un autre palier de gouvernement.

Il sera très difficile de trouver une solution globale. Il faudra faire preuve de beaucoup d'ingéniosité et de bonne volonté. Il en faudra peut-être plus que nous en possédons. Toutefois, la tâche sera totalement impossible si nous continuons à décrire le problème dans le vocabulaire trompeur et souvent démagogique de l'égalité par rapport à l'inégalité des provinces. Sous cet angle, le problème n'en est pas un, et l'importance actuelle de cette formulation témoigne d'un manque de lucidité et du déclin de la bonne volonté. Cela fait ressortir la méfiance mutuelle profonde qui est venue assombrir notre vie politique.

La partie de bras de fer constitutionnel à plusieurs dimensions qui se joue au Canada depuis quelques années n'a fait qu'empirer notre situation, en partie par la création et le renforcement de liens malsains. Il s'ensuit que des aspirations qui sont en elles-mêmes parfaitement compatibles en viennent à être considérées comme tragiquement opposées. Par exemple, on peut citer les liens entre la dualité linguistique et le multiculturalisme, ou ceux entre les autochtones et les Québécois, ou encore ceux entre l'égalité régionale et la société distincte. Il est peut-être déjà trop tard pour se défaire du ressentiment et de la méfiance, et il faudra une 
direction courageuse et clairvoyante pour y parvenir s'il en est encore temps. Il faudra aussi que nous voyions les aspirations de chacun telles qu'elles sont, libérées autant que possible de la rhétorique du mépris.

\section{NIVEAUX DE DIVERSITÉ}

Diverses solutions peuvent être entrevues au-delà de l'impasse où nous nous trouvons toujours. Une première voie serait fondée sur un dualisme dans lequel le Québec ne serait plus une unité fédérale comme les autres. L'autre gamme de solutions possibles serait basée sur un fédéralisme à quatre ou cinq régions, assez décentralisé pour permettre au Québec d'en être au même titre que les autres. L'une ou l'autre de ces solutions devrait s'adapter aux différences d'une façon que nous n'avons pas encore réussi à déterminer - du moins ouvertement et explicitement.

Pouvons-nous y arriver ? Il appert que non, mais j'aimerais néanmoins conclure en disant quelques mots sur ce que cela présupposerait.

D'une certaine façon, le fait de s'adapter aux différences définit ce qu'est le Canada. Bon nombre de Canadiens seraient d'accord sur ce point. C'est pourquoi la crise actuelle, faite de méfiance réciproque et de mauvaise volonté dans le débat constitutionnel, est si pénible à vivre pour beaucoup de nos compatriotes. Il n'y a pas seulement le fait que les deux sources de différence que j'ai décrites deviennent de plus en plus évidentes. On peut ramener d'anciennes questions sur le tapis. Dans une certaine mesure, la réalisation remarquable de Trudeau en matière de propagation du bilinguisme a été rendue possible par une sympathie grandissante envers le fait français parmi l'élite politique et sociale au CHQ. L'élite a accéléré l'implantation du bilinguisme beaucoup plus que ne l'auraient voulu certains concitoyens. Pour beaucoup de gens d'un palier inférieur dans la hiérarchie, le français leur était imposé. Étant donné l'influence de l'élite sur le processus de consentement politique du pays, ils n'ont pas eu d'autre choix que de l'accepter.

Au cours du débat à propos de l'accord du lac Meech, les procédures de négociation de cette élite ont été soumises à de sévères critiques et à la contestation. 
De plus, les élites du CHQ étaient également divisées entre elles quant à la façon de répondre au nouveau concept, chose qui ne s'était pas produite pour le bilinguisme. Il n'est donc pas surprenant qu'on ait commencé à se rebiffer contre le traitement offert au français. Voilà peut-être le signe avant-coureur d'une plus grande opposition à venir. On entend déjà les gens de l'Ouest dire que la dualité canadienne ne s'applique pas à eux, qu'ils vivent plutôt au sein d'une mosaïque multiculturelle. Les fondements d'une fédération bilingue se mettent de nouveau à vaciller. Cet axe fondamental de notre différence est menacé.

Fait encore plus essentiel, notre conception de la diversité est contestée. Parmi ceux qui se rallient à la Charte et au multiculturalisme pour rejeter la notion de société distincte, beaucoup sont fiers de leur ouverture à la diversité. Sur certains points, ils ont raison de l'être. Ce qui se trouve enchâssé dans ce texte, c'est ce qu'on pourrait appeler la diversité de premier degré. Il existe de grandes différences en matière de culture, de perspective et d'histoire chez une population qui n'en partage pas moins la même idée sur ce que signifie l'allégeance au Canada. Leur patriotisme ou mode d'appartenance est uniforme, peu importe leurs autres différences, et on considère que c'est nécessaire pour garder le pays uni.

Cette attitude est loin de plaire à tous les Canadiens. Pour les Québécois, et pour la plupart des Canadiens français, être canadiens (pour ceux qui veulent encore l'être) veut dire appartenir à un élément particulier du Canada, la nation québécoise ou canadienne-française. La même remarque s'applique grosso modo aux communautés autochtones du pays. La diversité du premier degré ne répond pas à leur façon d'être canadiens. Pourtant, bon nombre de personnes au CHQ restent perplexes quand ces groupes affirment se sentir exclus, parce que la diversité au premier degré est la seule à laquelle ils sont sensibles et qu'ils pensent connaître correctement.

Pour bâtir un pays ouvert à tous, le Canada devrait permettre l'existence de la diversité du deuxième degré ou diversité profonde, au sein de laquelle une pluralité de modes d'appartenance serait alors reconnue et acceptée. Un Torontois d'origine italienne, par exemple, ou encore un citoyen d'Edmonton d'origine ukrainienne pourraient bien se sentir canadiens à titre de titulaires de droits individuels dans une mosaïque multiculturelle. [82] Leur appartenance ne passerait pas par une autre communauté, bien que l'identité ethnique puisse être importante pour eux de diverses façons. Toutefois, ces personnes pourraient accepter qu'un Qué- 
bécois, un Cri ou un Déné conçoivent leur appartenance autrement que ceux qui se perçoivent canadiens tout en étant membres de leur communauté culturelle. Réciproquement, le Québécois, le Cri ou le Déné pourraient reconnaître la légitimité parfaite de l'identité selon le modèle de la mosaïque.

Cette perspective est-elle utopique ? Les gens pourraient-ils en venir à voir leur pays de cette façon ? Pourraient-ils trouver passionnant d'appartenir à un pays qui laisse place à la diversité profonde ? Pourraient-ils y trouver une certaine fierté ? Les pessimistes disent que non parce qu'ils ne voient pas comment ce pays pourrait avoir un sentiment d'unité. Le modèle de citoyenneté doit être uniforme ou les gens n'auront pas le sentiment d'appartenir au même régime. Ceux qui disent cela tendent à prendre les États-Unis comme modèle. Or, nos voisins sont opposés à la diversité profonde et l'ont même parfois qualifiée «d'antiaméricaine ».

Ces pessimistes devraient tenir compte de trois éléments. D'abord, c'est uniquement à partir de la diversité profonde qu'on peut reconstruire un Canada fédéral uni, une fois qu'on a rappelé les raisons pour lesquelles nous avons tous besoin du Canada - comme celles qui ont été citées ci-dessus, c'est-à-dire l'ordre public, la disposition collective, l'égalité régionale et l'aide mutuelle.

Ensuite, un peu partout dans notre monde, les différences ressemblent en degré et en nature à celles qui règnent au Canada plutôt qu'aux États-Unis. Si un modèle de citoyenneté uniforme correspond davantage à l'image classique de l'État occidental libéral, il est aussi vrai qu'il constitue une camisole de force pour bon nombre de sociétés politiques. Le monde a besoin que d'autres modèles soient auréolés de légitimité, afin de permettre que des modes de cohabitation politique plus humains et moins contraignants existent. Plutôt que d'accepter la rupture au nom d'un modèle uniforme, nous nous ferions une faveur tout en servant les intérêts des autres si nous explorions la solution de la diversité profonde. Pour ceux qui apprécient que l'on accorde aux gens la liberté d'être eux-mêmes, cette solution constituerait un gain pour la civilisation.

Nous ne serions pas seuls à nous lancer dans une pareille exploration. Les observateurs de la scène européenne ont vu que la création de la Communauté européenne a laissé plus de liberté aux sociétés régionales - bretonnes, basques, cata- 
lanes -, lesquelles étaient auparavant menacées d'écrasement sous le poids de l'État national.

Enfin, si une rupture devait mener à la création de deux régimes à citoyenneté uniforme, les deux États successeurs finiraient par découvrir qu'ils ne sont pas parvenus à relever le défi posé par la diversité profonde ; car la seule façon d'être juste envers les populations autochtones consiste à adopter une attitude pluraliste. Le Québec, tout comme le CHQ, n'irait nulle part s'il essayait de se hisser à la hauteur des États-Unis, ou encore au niveau des États nationaux européens, dans l'échelle du chauvinisme. Pourquoi ne pas reconnaître cette réalité immédiatement et s'engager ensemble sur la route de la diversité profonde?

\section{BIBLIOGRAPHIE}

ACKERMAN, Bruce, 1980, Social Justice in the Liberal State, New Haven, CT, Yale University Press.

CAIRNS, Alan, C., 1990a, "Constitutional Minoritarianism in Canada » dans Ronald WATTS et Douglas BROWN (dir.), Canada : The State of the Federation, 1990, Kingston, Institut des relations intergouvernementales, p. 71-96.

CAIRNS, Alan, C., 1990b, « Ritual, Taboo and Bias in Constitutional Controversies in Canada, or Constitutional Talk Canadian Style » dans The Saskatchewan Law Review, vol. 54, p. 121-147.

DWORKIN, Ronald, 1977, Taking Rights Seriously, London, Duckworth.

DWORKIN, Ronald, 1978, «Liberalism » dans Stuart HAMPSHIRE (dir.), Public and Private Morality, Cambridge, Cambridge University Press.

KYMLICKA, Will, 1989, Liberalism, Community and Culture, Oxford, Clarendon Press.

RAWLS, John, 1971, A Theory of Justice, Cambridge, MA, Harvard University Press. 
RAWLS, John, 1985, « Justice as Fairness : Political not Metaphysical » dans Philosophy and Public Affairs, vol. 14.

SANDEL, Michael, 1984, «The Procedural Republic and the Unencumbered Self » dans Political Theory, vol. 12.

TRIBE, Lawrence, 1990, Abortion : The Clash of Absolutes, New York, Norton.

TRUDEAU, Pierre Elliott, 1956, «La province de Québec au moment de la grève » dans P. E. TRUDEAU (dir.), La Grève de l'amiante, Montréal, Cité libre.

\section{Fin du texte}

